\title{
Evolution of VIM-1-Producing Klebsiella pneumoniae Isolates from a Hospital Outbreak Reveals the Genetic Bases of the Loss of the Urease-Positive Identification Character
}

\author{
Nicolas Cabanel, a,b Isabelle Rosinski-Chupin, ${ }^{\mathrm{a}, \mathrm{b}}$ Adriana Chiarelli, a,b,c Tatiana Botin,, ${ }^{\mathrm{a}, \mathrm{b}}$ Marta Tato, ${ }^{\mathrm{d}}$ (D) Rafael Canton, \\ (D) Philippe Glasera,b \\ aEERA Unit "Ecology and Evolution of Antibiotics Resistance," Institut Pasteur-Assistance Publique/Hôpitaux de Paris-Université Paris-Saclay, Paris, France \\ bUMR CNRS 3525, Paris, France \\ cSorbonne Université, Paris, France \\ đServicio de Microbiología, Hospital Universitario Ramón y Cajal and Instituto Ramón y Cajal de Investigación Sanitaria (IRYCIS), Madrid, Spain
}

Isabelle Rosinski-Chupin, Adriana Chiarelli, and Tatiana Botin contributed equally to this work.

ABSTRACT Outbreaks of carbapenemase-producing Klebsiella pneumoniae (CPKp) represent a major threat for hospitals. We molecularly characterized the first outbreak of VIM-1-producing $K$. pneumoniae in Spain, which raised fears about the spread of this strain or of the plasmid carrying $b / a_{\mathrm{VIM}-1}$. Through in-depth genomic analysis of 18 isolates recovered between October 2005 and September 2007, we show that 17 ST39 isolates were clonal, whereas the last isolate had acquired the VIM-1 plasmid from the epidemic clone. The index isolate carried 31 antibiotic resistance genes (ARGs) and was resistant to almost all antibiotics tested. Later isolates further gained mutations in efflux pump regulators ramR and opxR, deletion of $m g r B$ (colistin resistance), and frameshift mutations in ompK36 ( $\beta$-lactam resistance) likely selected by antibiotic usage. Comparison with publicly available genome sequences and literature review revealed no sign of dissemination of this CPKp strain. However, the VIM-1 plasmid was found in diverse Enterobacterales species, although restricted to Spain. One isolate became urease negative following IS5075 transposition into ureC. Analysis of 9,755 K. pneumoniae genomes showed the same ureC::IS5075 insertion in $14.1 \%$ of the isolates and explained why urease activity is a variable identification trait for $K$. pneumoniae. Transposition into ureC results from the similarity of its $3^{\prime}$ end and the terminal inverted repeats of Tn21-like transposons, the targets of IS5075 and related insertion sequences (ISs). As these transposons frequently carry ARGs, this might explain the frequent chromosomal invasion by these ISs and ureC inactivation in multidrug-resistant isolates.

IMPORTANCE Evolution of multidrug-resistant bacterial pathogens occurs at multiple scales, in the patient, locally in the hospital, or more globally. Some mutations or gene acquisitions, for instance in response to antibiotic treatment, may be restricted to a single patient due to their high fitness cost. However, some events are more general. By analyzing the evolution of a hospital-acquired multidrug-resistant $K$. pneumoniae strain producing the carbapenemase VIM-1, we showed a likely environmental source in the hospital and identified mutations contributing to a further decrease in antibiotic susceptibility. By combining the genomic analysis of this outbreak with literature data and genome sequences available in databases, we showed that the VIM-1 plasmid has been acquired by different Enterobacterales but is endemic only in Spain. We also discovered that urease loss in $K$. pneumoniae results from the specific transposition of an IS element into the ureC gene and was more frequent in fluoroquinolone-resistant isolates and those carrying a carbapenemase gene.
Citation Cabanel N, Rosinski-Chupin I, Chiarelli A, Botin T, Tato M, Canton R, Glaser P. 2021. Evolution of VIM-1-producing Klebsiella pneumoniae isolates from a hospital outbreak reveals the genetic bases of the loss of the urease-positive identification character mSystems 6:e00244-21. https://doi.org/10 $.1128 /$ mSystems.00244-21. Editor Robert G. Beiko, Dalhousie University Copyright $\odot 2021$ Cabanel et al. This is an open-access article distributed under the terms of the Creative Commons Attribution 4.0 International license. Address correspondence to Philippe Glaser, pglaser@pasteur.fr.

Received 1 March 2021

Accepted 6 May 2021

Published 1 June 2021 
KEYWORDS carbapenemase, insertion sequence, Klebsiella pneumoniae, drug resistance evolution, mobile genetic elements, urease

lebsiella pneumoniae is responsible for a broad range of diseases including pneumonia and bloodstream and urinary tract infections, mostly in health care facilities. $K$. pneumoniae isolates are frequently resistant to multiple antibiotics and contribute to the dissemination of antibiotic resistance genes (ARGs) $(1,2)$. Carbapenems are among the last-resort drugs to treat infections due to multidrug-resistant (MDR) Enterobacterales, including $K$. pneumoniae isolates expressing extended-spectrum $\beta$-lactamases (ESBLs). From the end of the 20th century onward, the emergence and dissemination of carbapenemase-producing $K$. pneumoniae (CPKp) resulting in high mortality rates have become a major public health threat. CPKp hospital outbreaks are particularly feared due to potential patient-to-patient transmission or transmission from the hospital environment to the patient. Recently, a broad genomic study on CPKp from 244 hospitals in 32 countries across Europe confirmed the existence of dominant lineages responsible for hospital outbreaks (3). In this study, the most prevalent multilocus sequence typing (MLST) types (STs) were from the clonal group (CG) 258, including ST258, -512, -340, -437, and -11, expressing the carbapenemase KPC $(1,3)$. Other prominent CPKp STs are ST307 (4) and ST101 (5). However, the molecular epidemiology of CPKp is different between countries (6), and a large proportion of CPKp isolates belongs to diverse and rare STs denoting relevance of local epidemiology.

In 2007, we reported the first case of a hospital outbreak involving CPKp isolates producing the VIM-1 carbapenemase in a hospital in Madrid, Spain $(7,8)$. During the same period, Escherichia coli, Klebsiella oxytoca, and Enterobacter cloacae isolates also producing VIM-1 were identified in the same hospital (7). Pulsed-field gel electrophoresis (PFGE) of K. pneumoniae isolates showed that they were likely clonal (8). This observation raised questions about the risk of endemicity of this clone and of the plasmid carrying bla $a_{\mathrm{VIM}-1}(7)$.

Whole-genome sequencing (WGS) is becoming instrumental to decipher hospital outbreaks and to characterize transmission (9). Point mutations and small indels, particularly those leading to gene inactivation or contributing to antibiotic resistance, are the main focus of genomic epidemiology studies. Other events, and in particular the mobility of insertion sequences (ISs), more difficult to identify by short-read sequencing, are frequently set aside. In this work, we have analyzed the evolution of the VIM-1producing $K$. pneumoniae isolates from the outbreak $(7,8)$. In addition to mutations selected by antibiotics used in the hospital, we observed a diversity in ARGs and plasmid contents and mobility of transposable elements: a group 2 intron and three ISs, IS26, IS5075, and IS421. In one isolate, IS5075 transposed into the ure operon encoding the urease subunits and led to a urease-defective phenotype. By analyzing 9,755 publicly available $K$. pneumoniae genome sequences, we show that this insertion is frequent, explaining why some $K$. pneumoniae isolates display a urease-negative phenotype. Furthermore, through a literature survey and the analysis of publicly available genome sequences, we did not find any evidence of further dissemination of this VIM1-producing strain. On the other hand, the $b / a_{\mathrm{VIM}-1}$ plasmid has broadly disseminated across Enterobacterales species but so far has been isolated only in Spain.

\section{RESULTS}

Genomic characterization of the outbreak isolates. Illumina WGS of the 18 isolates and in silico MLST showed that the first 17 isolates $\left(\mathrm{KP}_{\mathrm{VIM}} 1\right.$ to -17$)$ sharing the same PFGE profile belong to ST39 and the last isolate $\left(\mathrm{KP}_{\mathrm{VIM}} 18\right)$ belongs to ST45 (see Table S1 in the supplemental material). ST45 represents $1.5 \%(n=161)$ of the 10,515 genomes retrieved from NCBI (July 2020). ST39 is less frequent, with only 38 other genome sequences, including seven isolates carrying carbapenemase genes $\left(\right.$ bla $_{\mathrm{KPC}-3}$, $\left.n=3 ; b l a_{\mathrm{KPC}-2}, n=2 ; b l a_{\mathrm{NDM}-1}, n=2\right)$ but none carrying $b / a_{\mathrm{VIM}-1}$. In order to characterize the strain responsible for the outbreak and to identify genetic events occurring during its 


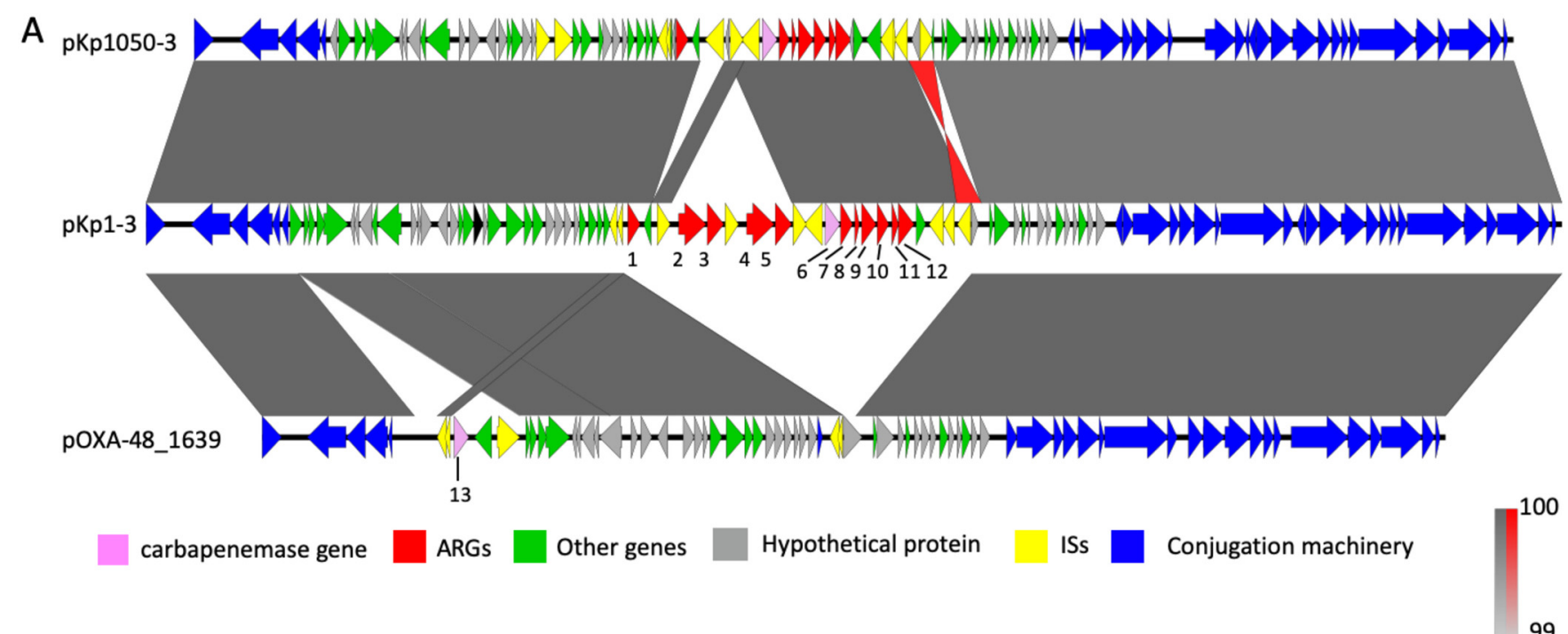

B

\begin{tabular}{|l|l|l|l|l|l|l|l|}
\hline Position (pKp1-3) & 11114 & 1937 & 15148 & 49053 & 50205 & 61124 & 75189 \\
\hline pKp1-3 & Mut. & \multicolumn{1}{|c|}{ Serratia marcescens, SCH909 plasmid pSCH909 (CP063239.1) } \\
\hline pOXA-48_1639 & \multicolumn{6}{|c|}{ pOXA-48 plasmids } \\
\hline
\end{tabular}

$\%$ similarity

FIG 1 Comparison of pKP1-3, pKP1050-3, and pOXA-48. (A) Comparison of plasmids pKP1-3 and pKP1050-3 (accession no. CP023419.1) carrying bla $a_{\mathrm{VIM}-1}$ and of pOXA-48_1639 carrying bla $a_{\text {OXA-48 }}$ (accession no. LR025105.1). pOXA48_1639 was chosen as it was the closest relative to pKP1-3. Gray areas between open reading frames denote nucleotide identities with a gradient representing $99 \%$ (light gray) to $100 \%$ (dark gray) identity. Identities of an inverted region are represented in red. Genes are indicated by arrows with a color code as in the figure key. Antibiotic resistance genes are numbered as follows: 1 , catA1; 2

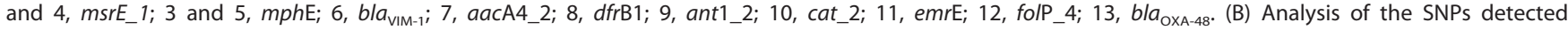
between pKP1-3 and pOXA-48_1639. Occurrences of SNPs among publicly available IncL/M plasmids ware identified by BLASTN. SNP positions in pKP1-3 are indicated in the first line. Mut. indicates that the mutation is specific to IncL/M VIM-1 plasmids. For other positions, plasmids with the pKP1-3 allele or the POXA-48_1639 allele are indicated in the second and third line, respectively. pSCH909 carries $b / a_{\mathrm{OXA}-10}$ and $b / a_{\mathrm{TEM}-1}$ but no carbapenemase gene.

evolution, we determined the complete genome sequence of the first isolate, $\mathrm{KP}_{\mathrm{VIM}} 1$. The $\mathrm{KP}_{\mathrm{VIM}} 1$ chromosome is $5,351,626$ bp long. It hosts four plasmids of 227,556 bp (pKP1-1), 110,924 bp (pKP1-2), 76,065 bp (pKP1-3), and 80,027 bp (pKP1-4) (Table S2). The chromosome and plasmids pKP1-1, -2 , and -3 carry 31 ARGs, including three in two copies [msr $(\mathrm{E}), m p h(\mathrm{E})$, and sul] (Table S2). Those ARGs target all major classes of antibiotics used against Gram-negative bacteria. The porin gene ompK35 is interrupted by a nonsense mutation at codon 230. In agreement with the ARG content, $\mathrm{KP}_{\mathrm{VIM}} 1$ is highly resistant to almost all antibiotics tested, remaining susceptible to only ciprofloxacin, tigecycline, imipenem, and colistin and exhibiting an intermediate phenotype to amikacin, nalidixic acid, meropenem, and ertapenem (Fig. S1).

The $b l a_{\mathrm{VIM}-1}$ gene is carried by a gene cassette inserted in a type 1 integron expressing six ARGs in addition to bla $a_{\mathrm{VIM}-1}$ (aacA4, dfrB1, ant1, cat, emrE, and folP_4) carried by plasmid pKP1-3 (Fig. 1). BLASTN search using the nucleotide sequence of this plasmid against the contigs of $\mathrm{KP}_{\mathrm{VIM}} 18$ showed $100 \%$ identity over its entire length, except a 1,722-bp region containing a catA gene and missing in $\mathrm{KP}_{\mathrm{VIM}} 18$. The VIM-1 plasmid was therefore likely transferred in the hospital from the outbreak strain to the ST45 K. pneumoniae isolate. Plasmid pKP1-3 belongs to the IncL/M type. Comparison with complete plasmid sequences showed that pKP1-3 is more than $99.9 \%$ identical over $89 \%$ of its length to pKP1050-3b carrying bla $a_{V / M-1}$ from a pan-drug-resistant $K$. pneumoniae strain isolated in June 2016 in a hospital in Madrid (Fig. 1) (10). Both plasmids are highly similar to a bla $a_{\mathrm{VIM}-1}$-carrying plasmid from a Salmonella enterica serovar Typhimurium strain isolated in Spain in 2014 (11) and from Klebsiella oxytoca strains isolated in Madrid in 2016 (12). Recently, a closely related plasmid was identified in 28 Serratia marcescens VIM-1-producing isolates recovered in our hospital as $\mathrm{KP}_{\text {VIM }} 1$ between September 2016 and December 2018 (13). We identified by BLASTN search 10 additional K. pneumoniae 


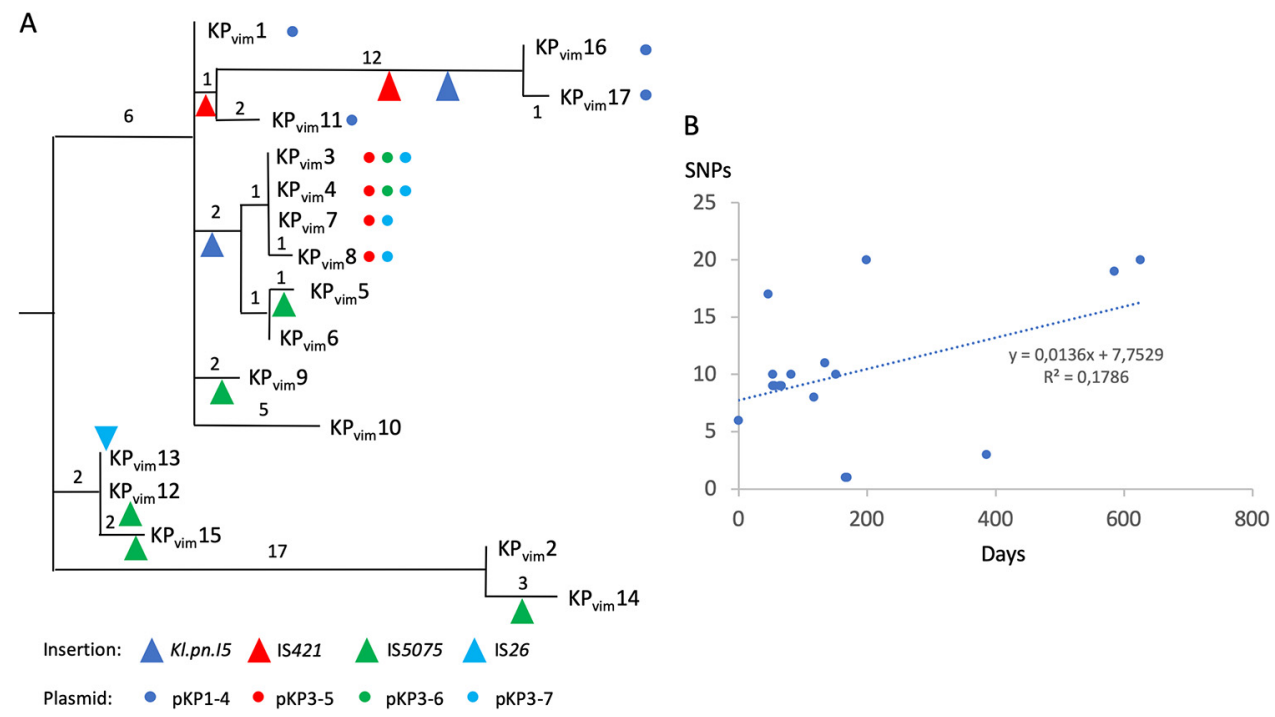

FIG 2 Hospital evolution of the K. pneumoniae ST39 VIM-1-producing strain. (A) Phylogeny of the 17 isolates reconstructed by maximum parsimony. Numbers next to branches indicate the number of chromosomal SNPs in the corresponding branch. Presence of plasmids is indicated by colored points, and transposition events are indicated by triangles. IS26 insertion in oqxR occurred in the common ancestor of $\mathrm{KP}_{\mathrm{VIM}} 12$ and $\mathrm{KP}_{\mathrm{VIM}} 13$. (B) Root-to-tip representation of the number of chromosomal SNPs according to the time (in days) following the isolation of the first isolate, $\mathrm{KP}_{\mathrm{VIM}} 1$. The trendline equation and the correlation coefficient are indicated on the graph.

isolates carrying a plasmid closely related to pKP1-3, among the $85 \mathrm{~K}$. pneumoniae genome sequences containing $b a_{\mathrm{VIM}-1}$ of the $10,515 \mathrm{~K}$. pneumoniae genome sequences from the NCBI (Table S3). Strikingly, these isolates from four different STs were also all isolated in Spain between 2010 and 2016. Therefore, IncL/M plasmids carrying $b / a_{\mathrm{VIM}-1}$ likely arose in Spain following the insertion of a type 2 integron and disseminated locally only but were recurrently isolated in Spain between 2005 and 2018.

These plasmids are closely related to the broadly distributed IncL/M plasmid pOXA48 carrying the bla $a_{\text {OXA-48 }}$ carbapenemase gene (10) (Fig. 1). pKP1-3 shows only seven single nucleotide polymorphisms (SNPs) over 57,386 conserved bp with pOXA48_1639, the closest relative identified at the NCBI (accession number LR025105.1). BLASTN search against the NCBI database showed that one SNP was specific to all characterized IncL/M VIM-1 plasmids, whereas for the six other positions, two different allelic forms could be identified: one shared by pOXA-48_1639 and other pOXA-48 plasmids, the other by pKP1-3 and IncL/M plasmids carrying other resistance genes. Therefore, these two plasmids share a very recent common ancestor which acquired either Tn1999 (14) carrying bla $a_{\mathrm{OXA}-48}$ or an integron carrying bla $a_{\mathrm{VIM}-1}$.

Intrahospital evolution of the ST39 lineage follows different paths associated with modifications of antibiotic susceptibility. On the basis of the variants identified, we reconstructed the evolutionary path of the 17 ST39 isolates (Fig. 2A). In total, we identified 64 SNPs (59 in the chromosome and five in the plasmids) and seven short indels, five of them leading to a frameshift in coding frames (Table S4). Ancestral genotype for each polymorphism was predicted by parsimony based on BLASTN comparisons with complete $K$. pneumoniae genome sequences at the NCBI. The first isolate, $\mathrm{KP}_{\mathrm{VIM}} 1$, shows six SNPs compared to the reconstructed sequence of the last common ancestor (LCA) of the 17 isolates. We next analyzed the root to tip number of chromosomal SNPs according to the time of isolation. Despite the duration of the outbreak over 24 months, we did not observe a strong temporal correlation (Fig. 2B).

We identified three large chromosomal deletions: a $6.3-\mathrm{kb}$ deletion encompassing mgrB, a 600-bp deletion of a type 6 secretion system (T6SS) immunity phospholipase A1-binding lipoprotein, and a 55.4-kb deletion corresponding to the excision of an integrated and conjugative element. Five large deletions in pKP1-1 and pKP1-2 led to 
A

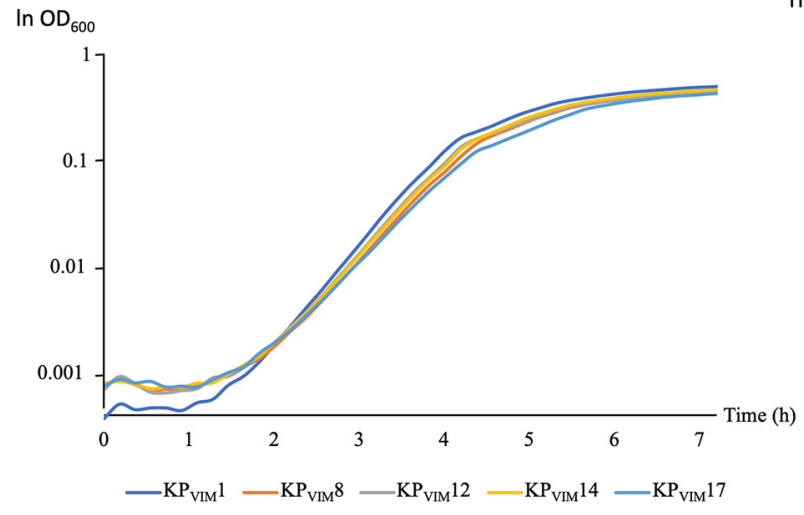

B

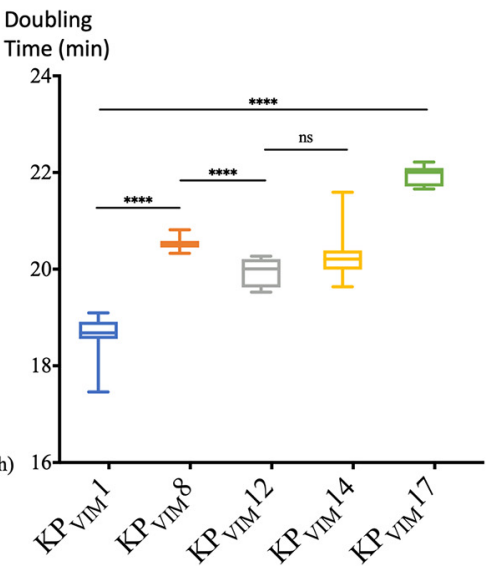

FIG 3 Growth and generation times of isolates with decreased antibiotic susceptibility. (A) Growth of $\mathrm{KP}_{\mathrm{VIM}} 1$ and of four isolates mutated in a repressor of efflux pumps $\left(\mathrm{KP}_{\mathrm{VIM}} 8, \mathrm{KP}_{\mathrm{VIM}} 12\right.$, and $\left.\mathrm{KP}_{\mathrm{VIM}} 14\right)$ or in $\mathrm{mgrB}$ and ompK36 (KP $\mathrm{VIM}_{17}$ 17) was followed by using an automatic plate reader. Background was subtracted as described in Materials and Methods. During the first $90 \mathrm{~min}$, the $\mathrm{OD}_{600}$ was below 0.0015 , and its quantification is noisy. (B) Box plot representation for 10 replicates of the generation times of the five isolates quantified in early exponential phase $2.5 \mathrm{~h}$ following the start of the culture $\left(\mathrm{OD}_{600}\right.$ between 0.005 and 0.04$)$. Statistical significances were tested with Student's $t$ test. ${ }^{* * *}, P \leq 0.0001 ; \mathrm{ns}$, nonsignificant.

the loss of clusters of ARGs (Table S2 and S4) in agreement with modifications of the antibiotic susceptibility profiles (Fig. S1).

Several genetic events were likely selected in response to antibiotic use in the hospital. The deletion of the $m g r B$ gene led to colistin resistance in $\mathrm{KP}_{\mathrm{VIM}} 17$ (Fig. S1). The same isolate was highly resistant to all $\beta$-lactams including carbapenems due to the inactivation of the second major porin gene, ompK36, by a nonsense mutation leading to a stop codon at position 125. In addition, we identified three mutations disrupting oqxR and ramR genes encoding repressors of efflux systems. oqx $R$ was inactivated by an IS26 insertion in $\mathrm{KP}_{\mathrm{VIM}} 12$ and $\mathrm{KP}_{\mathrm{VIM}} 13$, whereas ramR was inactivated by a nonsense mutation in $\mathrm{KP}_{\mathrm{VIM}} 14$ and by a frameshift mutation in $\mathrm{KP}_{\mathrm{VIM}} 7$ and $\mathrm{KP}_{\mathrm{VIM}} 8$. In agreement with previous comparisons of mutants of oqxR and ramR (15-18), we observed a stronger decrease in the susceptibility to fluoroquinolones in the isolates mutated in oqxR $\left(\mathrm{KP}_{\mathrm{VIM}} 12\right.$ and $\left.\mathrm{KP}_{\mathrm{VIM}} 13\right)$ and a stronger decrease in tigecycline susceptibility in the isolates mutated in ramR $\left(\mathrm{KP}_{\mathrm{VIM}} 7,-8\right.$, and -14). In the case of $\mathrm{KP}_{\mathrm{VIM}} 14$, the mutation in ramR likely compensates the loss of the qnrA1 gene for fluoroquinolone susceptibility. The five isolates also showed a decreased susceptibility to cefepime and cefoxitin (Fig. S1). To assess if there was any fitness cost associated with the increased resistance observed, we followed bacterial growth of these isolates in $L B$ at $37^{\circ} \mathrm{C}$. We observed in all four mutated isolates a decreased growth rate compared to $\mathrm{KP}_{\mathrm{VIM}} 1$. The effect was more pronounced for $\mathrm{Kp}_{\mathrm{VIM}} 17$ defective in both $\mathrm{mgrB}$ and ompK36, which showed a 17\% increase of generation time (Fig. 3).

Diversity of cryptic plasmid content. In the course of the epidemic strain evolution, we also observed changes in plasmid content (Fig. 2). Plasmid pKP1-4 is a IncFII type, which is present in the first isolate, $\mathrm{KP}_{\mathrm{VIM}} 1$, and in three of the last isolates of the outbreak ( $K P_{\mathrm{VIM}} 11,-16$, and -17$)$, reflecting its stability. This plasmid mainly codes for maintenance functions (toxin-antitoxin systems, colicin B production, and partition) and conjugative functions. BLASTN search against bacterial genome sequences showed that pKP1-4 is almost identical (99.7\% identities over its entire length) to plasmid pEC14III (accession number KU932028.1) from an E. coli strain isolated in Finland. We also identified three plasmids specific to the lineage $\mathrm{KP}_{\mathrm{VIM}} 3$ to $\mathrm{KP}_{\mathrm{VIM}} 8$ (Fig. 2). These six isolates share a 34,017-bp-long, linear plasmid (pKP3-5) flanked by two 695-bp-long terminal inverted repeats (TIRs). Unlike most linear plasmids described in K. pneumoniae, pKP3-5 is unrelated to phages. No adaptive functions were recognized, unlike in a 
A

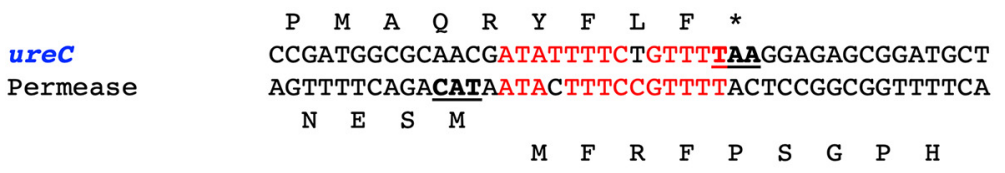

pKP1-3 EAL AGGCTTAACGTAGGATATTTTCCGTTTTCCAAGCGGCCCCCATA pKP1-2 Tn3-1ike CGGCTTAGCGTGCTTTATTTTCCGTTTTCTGAGGCGACCCCCAC pKP1-3 Tn3-1ike CGGCTTAGCGTGCTTTATTTTCCGTTTTCTGAGACGACCCCTAT Consensus

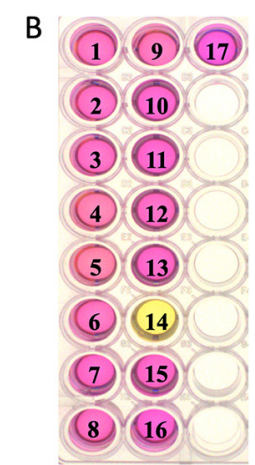

FIG 4 Urease inactivation following IS5075 transposition. (A) Sequence alignment of the sites targeted by IS5075 among $\mathrm{KP}_{\mathrm{VIM}}$ isolates. In blue, targets of transposition events occurring during the outbreak: ureC in $\mathrm{KP}_{\mathrm{VIM}} 14$ and pKP1-3 Tn21 in $\mathrm{KP}_{\mathrm{VIM}} 5, \mathrm{KP}_{\mathrm{VIM}}$ 9, $\mathrm{KP}_{\mathrm{VIM}} 12$, and $\mathrm{KP}_{\mathrm{VIM}} 15$. The green triangles correspond to IS5075 insertion sites. Conserved bases are indicated in red. Stop and start codons are underlined. (B) Urease activity test of the 17 ST39 isolates. The number of each $\mathrm{KP}_{\mathrm{VIM}}$ isolate is indicated on the well. A pink color of the indole reaction reveals a urease-positive phenotype.

similar linear plasmid, pBSSB1 from Salmonella enterica serovar Typhi, that encodes a flagellin structural gene (19). A search among K. pneumoniae genomes revealed 19 isolates carrying putative linear plasmids closely similar to pKP3-5 (>90\% identities over $90 \%$ of the length). The two other plasmids are small high-copy-number plasmids, pKP3-6 (2,811 bp) and pKP3-7 (3,861 bp), that are present in strains $\mathrm{KP}_{\mathrm{VIM}^{3}} 3$ to -6 and $\mathrm{KP}_{\mathrm{VIM}} 3$ to -8 , respectively (Fig. 2 and Table S2). No adaptive functions were predicted in these two plasmids. For these three plasmids, we could not determine whether they were gained in the common ancestor of the $\mathrm{KP}_{\mathrm{VIM}} 3$ to $\mathrm{KP}_{\mathrm{VIM}} 8$ clade or lost by other isolates.

Insertion of IS5075 into ureC is responsible for a urease-negative phenotype in one isolate of the outbreak. In addition to IS26 insertion in oqxR, we identified nine transpositions of mobile genetic elements: two insertions of a class 2 intron named KI.pn.I5 (20) and two and five transpositions of IS421 and IS5075, respectively (Fig. 2). Compared to the other isolates, $\mathrm{KP}_{\mathrm{VIM}} 14$ was characterized by an IS5075 inserted three codons upstream of the stop codon of the ureC gene encoding the urease catalytic subunit (Fig. 4A). This insertion led to a ureC-IS5075 transposase gene fusion. It might also have a polar effect on the expression of the downstream genes of the operon: ureE, ureF, and ureG. Accordingly, the $\mathrm{KP}_{\mathrm{VIM}} 14$ isolate was urease negative, whereas all other isolates from the outbreak were urease positive (Fig. 4B). IS5075, like its close relative IS4321, is known to transpose into the TIR of Tn21 and of related transposons of the Tn3 family (21). Tn3 family transposons are abundant and diverse (22). They are vectors of heavy metal resistance and ARGs (21). The 17 ST39 isolates harbor three copies of IS5075 inserted in a pKP1-2 Tn3 family transposon, just after the initiation codon of a pKP1-1 gene coding for an EAL motif protein and upstream of a chromosomal permease gene (Fig. 4A). Four independent and identical transposition events of IS5075 also occurred in the TIR of a Tn3 family transposon carried by PKP1-3, in $\mathrm{KP}_{\mathrm{VIM}} 5, \mathrm{KP}_{\mathrm{VIM}} 9$, $\mathrm{KP}_{\mathrm{VIM}} 12$, and $\mathrm{KP}_{\mathrm{VIM}} 15$ (Fig. $2 \mathrm{~A}$ ). Based on the conservation of the insertion sites of IS5075, we proposed a 13-bp consensus sequence for the IS5075 transposition site (Fig. 4A).

Urease-negative phenotypes are prevailing in several $K$. pneumoniae MDR lineages. Urea hydrolysis is an identification trait of $K$. pneumoniae in clinical microbiology laboratories. However, earlier reports have shown that $5 \%$ of K. pneumoniae isolates are urease negative (23). In order to determine whether this phenotype was due to similar IS5075 transposition, we analyzed the ureC gene in 9,755 K. pneumoniae genomes quality filtered from the 10,515 genome sequences retrieved from the NCBI (Table S5). BLASTN search showed that an IS5075 or a similar IS was inserted at the same position in 1,380 isolates $(14.1 \%)$ (Table 1 ). A search for other insertions or 
TABLE 1 Comparison of ureC::IS5075 and ureC WT K. pneumoniae isolates for antibiotic resistance features and ARG and IS copy numbers

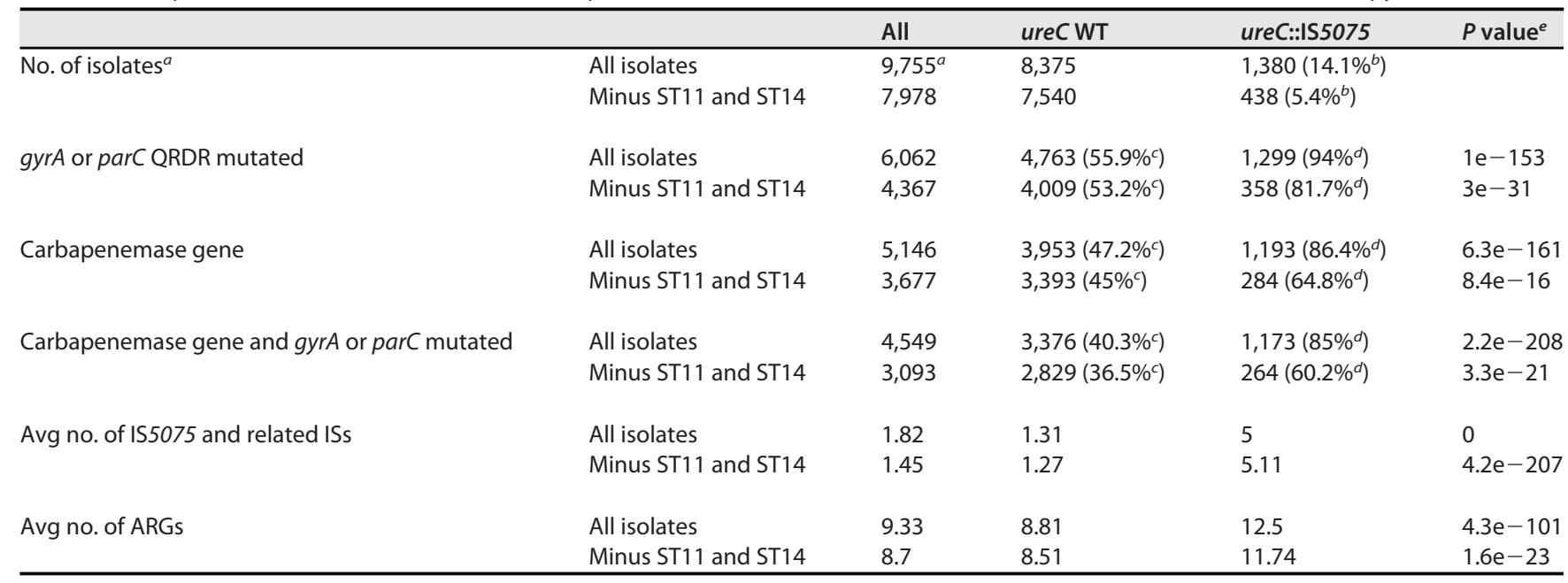

${ }^{a}$ After filtering out 760 genome sequences out of the 10,515 sequences retrieved from the NCBI.

bPercentage of isolates with an IS insertion in ureC.

cPercentage of ureC WT isolates mutated in QRDR and/or carrier of carbapenemase genes.

dPercentage of ureC::IS5075 isolates mutated in QRDR and/or carrier of carbapenemase genes.

eSignificance of the difference between ureC::IS5075 and ureC as determined by the chi-square or the Wilcoxon rank sum statistical test.

frameshifts in ureC did not reveal other frequent mutations putatively responsible for a urease deficiency.

To determine whether the insertion of IS5075 into ureC preferentially occurred under specific genetic backgrounds, we analyzed the $45 \mathrm{~K}$. pneumoniae STs with at least 20 isolates (Fig. 5). We observed that IS5075 urease inactivation occurred throughout the species with variable frequencies. In seven STs, all with fewer than 100 isolates, no insertion was observed. On the other hand, we observed a high proportion of ureC:: IS5075 isolates in some STs like ST11 (884 out of 1,603) and ST340 (18 out of 77 isolates) from the clonal group (CG) 258 and ST14 (58 out of 174). On the other hand, the two other dominant CG258 STs, ST258 and ST512, showed lower insertion frequencies of $6.9 \%$ and $4.1 \%$, respectively.

As several of the STs associated with a higher frequency of ureC::IS5075 include major MDR lineages, we next analyzed the distribution of IS insertions in ureC in relation to antibiotic resistance. As markers of antibiotic resistance, we considered mutations in fluoroquinolone resistance (FQR) determinants, presence of carbapenemase genes, and the number of ARGs among the 9,755 K. pneumoniae genome sequences (Table S5). Among these genome sequences, $62 \%$ were mutated in $g y r A$ and/or parC quinolone resistance-determining regions (QRDRs), 53\% carried carbapenemase genes, and the average number of ARGs was 9.33, revealing a strong bias toward MDR isolates (Table 1). Despite this bias, ureC::IS5075 isolates appeared as even more resistant, with an average number of 12.5 ARGs compared to 8.8 in the remaining isolates, $94 \%$ of the isolates showing mutations in $\operatorname{gyr} A$ and/or parC and $86.4 \%$ carrying a carbapenemase gene (Table 1). To determine whether the insertion in ureC was associated with a global expansion of IS5075 and related ISs, we estimated the copy number of these ISs in the different isolates (Table 1). Isolates with an IS insertion in ureC showed on average a 4-fold-higher copy number of IS5075 and related ISs than the remaining isolates ( 5 versus 1.31). On the other hand, more than half of the isolates with an intact ureC gene did not carry a single IS5075 copy (4,334 out of 8,375).

In a given ST, a high frequency of ureC::IS5075 isolates might result from frequent transposition events or from the expansion of lineages carrying the insertion. To discriminate between these two possibilities, we performed a whole-genome phylogeny focusing on ST11, ST14, and ST258. ST11 was the most abundant ST among the genome sequences retrieved from the NCBI (16.4\% of all isolates). Except two isolates 


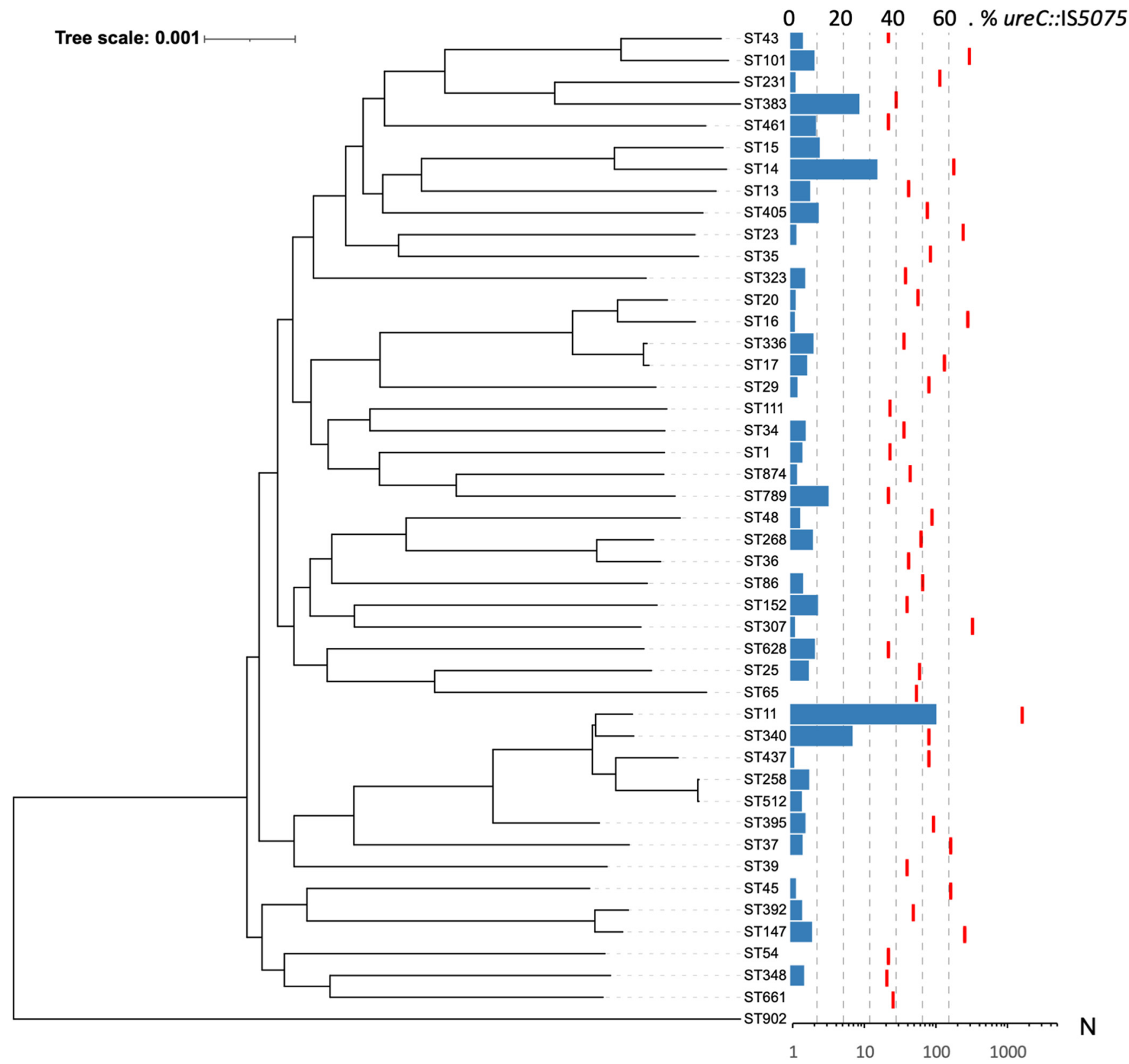

FIG 5 Distribution of IS5075 insertions in ureC among K. pneumoniae isolates. Occurrence of IS5075 insertion among the 45 STs with at least 20 isolates among 9,755 K. pneumoniae genome sequences retrieved from the NCBI. Phylogeny was reconstructed using Parsnp (50) and by using a representative isolate from each ST. The tree was rooted according to the work of David et al. (3). Blue bars indicate the percentage of isolates with an insertion in ureC (upper scale), and red dashes indicate the number of isolates in the corresponding ST (lower scale).

that were wild type (WT) for gyrA and parC, all ST11 isolates were predicted to be FQR (Fig. 6). The two most populated lineages belong to the K-types KL64 $(n=622)$ and $\mathrm{KL} 47(n=463)$. These closely related lineages share the same three mutations in QRDRs (ParC-80I, GyrA-83I, GyrA-87G) and carry the carbapenemase gene bla $a_{\mathrm{KPC}-2}$. Analysis of IS5075 insertions in ureC showed an uneven distribution, mostly associated with these two lineages. In the KL64 clade, the IS insertion is ancestral, as it was present in all except six isolates (in pink). In the KL47 clade, two different situations were noted: an ancestral transposition event in the LCA of a specific sublineage, with the 138 isolates from this clade showing an IS5075 in ureC (clade colored in red), and a relatively high frequency of insertion in the other isolates of the clade (85 out of 324, 26\%) likely 

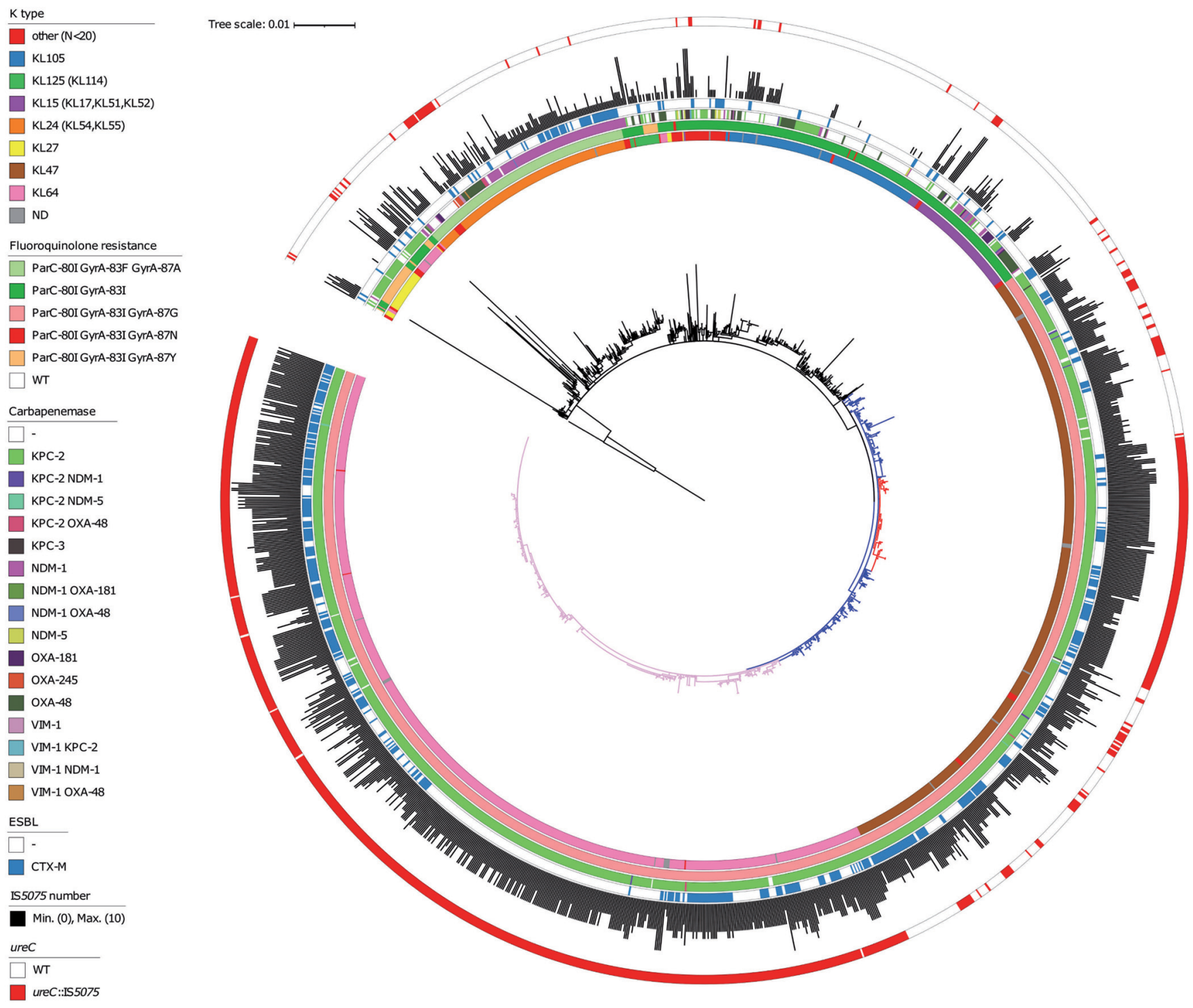

FIG 6 Core genome phylogeny of K. pneumoniae ST11 isolates. Phylogeny was obtained by using Parsnp (50) considering 1,603 genomes passing the quality threshold. K-type, mutations in gyrA and parC QRDRs, carbapenemase genes, bla $a_{\text {TX-m }}$ genes, copy number of IS5075 and related ISs, and IS insertion in ureC are annotated by circles from inside to outside as indicated in the figure key (left). The ureC-deficient KL64 lineage is in pink. The KL47 lineage is in blue, and the ureC-deficient sublineage is in red. The two gyrA/parC WT isolates were used as outgroups to root the tree. The tree was visualized by using iTOL (51). ND, no data.

resulting from multiple sporadic transposition events. Out of the two clades, the frequency of insertion is much lower (8.5\%). All over the ST11 phylogeny, insertion in the ureC gene was associated with a higher copy number of IS5075 with on average 5 copies compared to 1.7 in ST11 isolates with a WT ureC gene. Altogether, these results show that the high proportion of ST11 isolates mutated in ureC results in a large part from the dissemination of two clades showing a high number of IS5075 copies. The situation was similar among ST14 isolates, as all but one isolate $(n=58)$ mutated in ureC belonged to a single FQR lineage, suggesting that transposition occurred in the LCA of the lineage (in blue, Fig. S2). Isolates of this lineage also showed a high IS5075 copy number $(n=5.1)$. In ST258, isolates were characterized by a lower frequency of IS insertion in ureC (6.9\%). Most of the ST258 isolates cluster in two lineages expressing two different capsule operons of K-types KL106 and KL107 and associated mostly with the carbapenemase genes $b / a_{\mathrm{KPC}-2}$ and $b l a_{\mathrm{KPC}-3}$, respectively (24). In contrast to what was observed in ST11 and ST14, no expansion of a large ureC::IS5075 clade occurred 
(Fig. S3). All but two isolates with the insertion in ureC belonged to the KL107 lineage. Strikingly, this clade was characterized by a higher copy number of IS5075 of 2.17 (5.24 for ureC::IS5075 isolates) compared to only 0.12 for the KL106 lineage. Therefore, a major driver for insertion into ureC is the presence of an IS5075 or a related IS and its active transposition.

\section{DISCUSSION}

Whole-genome sequencing has revolutionized molecular epidemiology, and its use in outbreak analysis has contributed to deciphering the path of pathogen transmission (25). Here, we investigated the first outbreak due to a VIM-1-producing $K$. pneumoniae strain in Spain $(7,8)$. The strain was extensively drug resistant and belongs to an uncommon ST (ST39). Based on available genomic data, we showed that the strain preexisted in the hospital prior to the identification of the first isolate in October 2005. Furthermore, the weak temporal signal in the evolution (Fig. 2B) indicated a likely environmental reservoir in the hospital, which agrees with epidemiological data (7). Molecular clocks for K. pneumoniae evolution have been estimated between 1.4 (26), 1.9 (27), and 3.65 (28) mutations $/ 10^{6} \mathrm{bp} /$ year. Here, the rate of SNPs $/ 10^{6} \mathrm{bp} / \mathrm{year}$ is in the lower range $(n=0.87)$. Growth as a biofilm compared to planktonic growth has been related to a greater diversity due to its structured organization but a lower mutation rate due to a reduced number of generations (29). The diversity observed, the duration of the outbreak, and the small number of SNPs agree with a biofilm source of the isolates. In line with this observation, we observed biofilm production of all the isolates but to variable levels (see Fig. S4 in the supplemental material).

During the 2-year evolution of the strain, we observed variations in the antibiotic resistance profile. This was due on the one hand to the loss of ARGs (Table S4). On the other hand, mutations leading to the increased expression of efflux pumps or to a decreased drug permeation, and subsequently to a decreased susceptibility to some antibiotics, were selected. However, these mutations led to a fitness cost (Fig. 3), which might explain their limited expansion in the hospital.

By combining genomic analysis of the strain responsible for the outbreak with global genomic information retrieved from the NCBI and data from the literature, we were able to draw more general conclusions related to the risk associated with the outbreak strain and the VIM-1 plasmid. Likewise, we were able to identify the main reason for urease deficiency among $K$. pneumoniae isolates. Following the identification of the first VIM-1 isolates in Spain, their dissemination was a matter of concern (7). Although we showed that one single ST39 clone, except for one isolate, was responsible for the outbreak, we did not identify any new occurrence of this strain or of an ST39 isolate carrying $b / a_{\mathrm{VIM}-1}$ based on bibliographical survey and on the analysis of more than 10,000 publicly available $K$. pneumoniae genome sequences. Therefore, this clone seems to be restricted to the hospital where it was isolated. Conversely, we showed that the plasmid carrying $b / a_{\mathrm{VIM}-1}$ has disseminated among various Enterobacterales species. Transfers occurred probably in the hospital context, as suggested in the case of an S. Typhimurium isolate (11). Similarly, we showed the transmission of the VIM-1 plasmid between K. pneumoniae isolates in the course of the outbreak. We previously predicted similar transfers between $K$. pneumoniae and $E$. coli based on plasmid typing and size determination (7). This IncL/M plasmid is closely related to the broadly disseminated pOXA48. Our mutation analysis strongly suggests independent gain of a carbapenemase gene by very similar plasmid backbones showing only seven SNPs over $57,386 \mathrm{bp}$. In agreement with this hypothesis, the first OXA-48 plasmid was detected in Spain in 2009 (30), 4 years after the first VIM-1 isolate of the hospital outbreak (7).

Strikingly, until now only IncL/M VIM-1 plasmids were reported in Spain. A recent study on plasmids encoding VIM-1 from broad origins showed that among the 28 plasmids analyzed, nine were from IncL/M type (31). These nine plasmids were related to pKP1-3 and were from K. pneumoniae, Enterobacter hormaechei, and E. cloacae and all from Spain. The limited dissemination of the VIM-1 plasmid might be due to the 
conjunction of different factors including: a lower conjugation efficiency than pOXA-48 plasmids, a fitness cost restricting its dissemination to environments characterized by strong selective pressures, such as the hospital, or a specificity in antibiotic prescription in Spain. Comparing IncL/M VIM-1 and OXA-48 plasmids provides a model system to study two closely related plasmids with two different spreading destinies.

Urease is considered in many bacterial species as a virulence factor beyond its contribution in harnessing urea as a nitrogen source (32). Urease participates in the adaptation to acidic conditions in a broad range of human pathogens, including Helicobacter pylori (33), Yersinia enterocolitica (34), and Proteus mirabilis (35). Urease is considered a potential target for the development of new antibacterial drugs against enteric bacteria including K. pneumoniae (36). In K. pneumoniae, the urease has been shown to contribute to gastrointestinal colonization (37). However, a significant proportion of $K$. pneumoniae isolates are urease negative. Here, we showed that the inactivation of the operon is due to the transposition into the ureC gene of IS5075 or of related ISs, like IS4321, sharing the same specificity. Urease inactivation can be observed in both carriage isolates and isolates associated with clinical symptoms. For instance, we identified a cluster of eight IS5075::ureC ST340 isolates from a single institution (Fig. S5). These isolates were recovered from three patients, from urinary tract infections, blood culture, cerebrospinal fluid, and fecal carriage (38).

Among ureC::IS5075 isolates, we observed a higher prevalence of gyrA and parC mutations and of carbapenemase genes and, more generally, a higher number of ARGs compared to ure C WT isolates (Table 1). This was partly due to a small number of MDR lineages mutated in ureC, such as those of ST11 and ST14, which represent $68 \%$ of the ureC::IS5075 isolates (Fig. 6 and Fig. S2). Nevertheless, this higher prevalence remained true even after removing ST11 and ST14 isolates (Table 1). IS insertions in ureC were also associated with a 4-fold increase in IS5075 copies, resulting from additional transposition events (Table 1). This expansion of IS5075 in some genetic backgrounds might be a relatively recent event. Indeed, $44 \%$ of the isolates did not carry a single IS5075 copy, despite the high number of ARGs in the genomes we have analyzed. Indeed, the most frequent targets of IS5075 are the conserved TIR of transposons related to $\mathrm{Tn} 21$, which are ARG vectors and frequently carried by conjugative plasmids as in the case of pKP1-2 $(21,22)$. This insertion specificity represents a safe harbor for these ISs, as it does not incur fitness costs and ensures their dissemination. The insertion into $u$ reC results from the high similarity between its last codons and TIRs of Tn21 and is likely accidental. Therefore, the higher frequency of ureC inactivation in some MDR lineages might merely be a consequence of a more frequent acquisition of Tn3 family transposons carrying IS5075. However, we cannot completely dismiss the possibility that the loss of urease activity might provide MDR K. pneumoniae isolates with a selective advantage under some circumstances. This seems rather unlikely, as other ureC inactivation events, including transpositions of other ISs, would have been expected in that case and we did not detect such events. Overall, IS5075 transposition into the K. pneumoniae ureC gene represents a perfect example of chromosomal colonization by IS elements carried by plasmids and leading to a homoplasic loss of function.

\section{MATERIALS AND METHODS}

Bacterial strains, growth conditions, and antibiotic susceptibility testing. VIM-1-producing $K$. pneumoniae isolates were collected from 2005 through 2008 at Ramon y Cajal University Hospital in Madrid, Spain (8) (see Table S1 in the supplemental material). The colistin MIC was determined in Mueller-Hinton (MH) broth as recommended by the Clinical and Laboratory Standards Institute (CLSI) guidelines (39). Susceptibility to 33 other antibiotics (Fig. S1) was evaluated by disk diffusion on MH agar according to the CLSI guidelines (39). Fitness was determined by growth curve analysis with a Tecan Infinite M200 automatic spectrophotometer during $24 \mathrm{~h}$ in LB. Wells were inoculated with overnight cultures at an optical density at $600 \mathrm{~nm}\left(\mathrm{OD}_{600}\right)$ of $0.001 . \mathrm{OD}_{600}$ was measured every $10 \mathrm{~min}$. Background was determined as the average value of the $\mathrm{OD}_{600}$ of the first three time points. Doubling time was determined between $\mathrm{OD}_{600} \mathrm{~S}$ of 0.005 and 0.03 , where an almost perfect fit with an exponential growth was observed. 
Genome sequencing and sequence analysis. $K$. pneumoniae genomes were sequenced by using the Illumina HiSeq2500 platform, with 100-nucleotide (nt) paired-end reads. Libraries were constructed by using the Nextera XT kit (Illumina). Reads were assembled with SPAdes 3.9.0 (40). The complete genome sequence of strain $\mathrm{KP}_{\mathrm{VIM}} 1$ was determined by using the long-read PacBio technology (Macrogen, Seoul, South Korea). Reads were assembled with the RS_HGAP_Assembly.3 protocol (41) and with Canu (42). The consensus sequence was polished with Quiver (41) and manually corrected by mapping Illumina reads with Breseq 0.33.2 (43). Variants compared to $\mathrm{KP}_{\mathrm{VIM}} 1$ were identified by using Breseq (43). Genome sequences were annotated with Prokka 1.14.5 (44) and analyzed for MLST and ARG content by using Kleborate (45) and Resfinder 4.0.1 (46). Plasmid incompatibility groups were identified by using PlasmidFinder 2.1 (47). Directionality of mutations was determined as previously described by performing BLASTN comparisons against publicly available $K$. pneumoniae genomes (48).

Analysis of publicly available genome sequences. K. pneumoniae genome assemblies $(n=10,515)$ were downloaded from the NCBI (July 2020) with Batch Entrez (49). Genome sequences with more than 200 contigs of more than 500 nt were filtered out. Sixty genome sequences (BioProject PRJNA510003) for which the contig ends corresponding to repeated sequences have been trimmed were removed from the analysis. In total, we analyzed IS5075 insertions in 9,755 genome sequences (Table S5). Phylogenetic analysis was performed by using Parsnp 1.1.2 (50). Recombination regions were visually identified as regions with a higher SNP density by using Gingr (50) and removed from the reference genome sequence (ST11, strain FDAARGOS_444, CP023941.1; ST14, strain 11, CP016923.1; ST258, strain BIC-1, NZ_CP022573.1; ST340, strain EuSCAPE_RS081, GCA_902155965.1_18858_1_51). Insertion of IS5075 and of related ISs in ureC was identified by BLASTN search using as query sequence the junction sequence detected in the $\mathrm{KP}_{\mathrm{VIM}} 14$ isolate encompassing $20 \mathrm{nt}$ of the ureC gene and $20 \mathrm{nt}$ of IS5075 (E value of $1 \mathrm{e}-10$ as threshold). The integrity of ureC was tested by tBLASTn using the UreC protein sequence from $\mathrm{KP}_{\mathrm{VIM}} 1$ as query. Copy number of IS5075 and of closely related ISs was estimated by counting BLASTN hits (100\% identity over the entire length), using the first 17 nucleotides of the IS5075 sequence as query. Phylogenetic trees were visualized by using iTOL (51).

Phenotypic analyses. The urease detection test was carried out with urea-indole medium (Bio-Rad) according to the manufacturer's instructions. Biofilm formation capacity was measured by the microtiter plate assay as previously described (52). K. pneumoniae strain LM21 (53) was used as a positive control.

Statistical analysis. The significance of the differences in frequencies of IS insertions in ureC was determined by using the chi-square test. The significance of differences in IS5075 copy numbers and in ARG numbers was determined by the Wilcoxon rank sum test. Both tests were performed by using standard libraries contained within the R statistics package (http://www.R-project.org/). Statistical significances of growth rate differences were tested with a Student $t$ test.

Availability of data. All sequence data have been deposited at DDBJ/EMBL/GenBank (BioProject PRJEB41835) with the following accession numbers: LR991401, KP $\mathrm{VIM}_{1} 1$ chromosome and plasmids; LR991487, plasmid pKP1-5; LR991544, plasmid pKP1-6; LR991565, plasmid pKP1-7. BioSample identifiers for the Illumina sequence data are listed in Table S1.

\section{SUPPLEMENTAL MATERIAL}

Supplemental material is available online only.

FIG S1, PDF file, $0.1 \mathrm{MB}$.

FIG S2, PDF file, $0.2 \mathrm{MB}$.

FIG S3, PDF file, 0.7 MB.

FIG S4, PDF file, $0.1 \mathrm{MB}$.

FIG S5, PDF file, $0.1 \mathrm{MB}$.

TABLE S1, PDF file, $0.03 \mathrm{MB}$.

TABLE S2, PDF file, $0.03 \mathrm{MB}$.

TABLE S3, PDF file, $0.02 \mathrm{MB}$.

TABLE S4, XLSX file, $0.1 \mathrm{MB}$.

TABLE S5, XLS file, 3.7 MB.

\section{ACKNOWLEDGMENTS}

This work was supported by grants from the French National Research Agency (ANR10-LABX-62-IBEID), and from the European Union's Horizon 2020 Research and Innovation Program under grant agreement no. 773830 (Project MedVetKlebs, One Health EJP). Adriana Chiarelli is part of the Pasteur-Paris University (PPU) International PhD Program, with funding from the Institut Carnot Pasteur Microbes \& Santé, and the European Union's Horizon 2020 research and innovation program under the Marie Sklodowska-Curie grant agreement no. 665807.

We thank Rafael Patiño-Navarrete for his help in the bioinformatics analysis and Laurence Ma from the Institut Pasteur Biomics platform (C2RT, Institut Pasteur, Paris, France, supported by France Génomique, ANR-10-INBS-09-09 and IBISA) for her help in Illumina sequencing. 


\section{REFERENCES}

1. Wyres KL, Holt KE. 2018. Klebsiella pneumoniae as a key trafficker of drug resistance genes from environmental to clinically important bacteria. Curr Opin Microbiol 45:131-139. https://doi.org/10.1016/j.mib.2018.04.004.

2. Navon-Venezia S, Kondratyeva K, Carattoli A. 2017. Klebsiella pneumoniae: a major worldwide source and shuttle for antibiotic resistance. FEMS Microbiol Rev 41:252-275. https://doi.org/10.1093/femsre/fux013.

3. David S, Reuter S, Harris SR, Glasner C, Feltwell T, Argimon S, Abudahab K, Goater R, Giani T, Errico G, Aspbury M, Sjunnebo S, Feil EJ, Rossolini GM, Aanensen DM, Grundmann H. 2019. Epidemic of carbapenem-resistant Klebsiella pneumoniae in Europe is driven by nosocomial spread. Nat Microbiol 4:1919-1929. https://doi.org/10.1038/s41564-019-0492-8.

4. Wyres KL, Hawkey J, Hetland MAK, Fostervold A, Wick RR, Judd LM, Hamidian M, Howden BP, Lohr IH, Holt KE. 2019. Emergence and rapid global dissemination of CTX-M-15-associated Klebsiella pneumoniae strain ST307. J Antimicrob Chemother 74:577-581. https://doi.org/10.1093/jac/ dky492.

5. Roe CC, Vazquez AJ, Esposito EP, Zarrilli R, Sahl JW. 2019. Diversity, virulence, and antimicrobial resistance in isolates from the newly emerging Klebsiella pneumoniae ST101 lineage. Front Microbiol 10:542. https://doi .org/10.3389/fmicb.2019.00542.

6. Bonnin RA, Jousset AB, Chiarelli A, Emeraud C, Glaser P, Naas T, Dortet L. 2020. Emergence of new non-clonal group 258 high-risk clones among Klebsiella pneumoniae carbapenemase-producing $K$. pneumoniae isolates, France. Emerg Infect Dis 26:1212-1220. https://doi.org/10.3201/eid2606 .191517.

7. Tato M, Coque TM, Ruçz-Garbajosa P, Pintado V, Cobo J, Sader HS, Jones RN, Baquero F, Canton R. 2007. Complex clonal and plasmid epidemiology in the first outbreak of Enterobacteriaceae infection involving VIM-1 metallo-beta-lactamase in Spain: toward endemicity? Clin Infect Dis 45:1171-1178. https://doi.org/10.1086/522288.

8. Tato M, Morosini M, Garcia L, Alberti S, Coque MT, Canton R. 2010. Carbapenem heteroresistance in VIM-1-producing Klebsiella pneumoniae isolates belonging to the same clone: consequences for routine susceptibility testing. J Clin Microbiol 48:4089-4093. https://doi.org/10.1128/JCM $.01130-10$.

9. Snitkin ES, Zelazny AM, Thomas PJ, Stock F, Henderson DK, Palmore TN, Segre JA. 2012. Tracking a hospital outbreak of carbapenem-resistant Klebsiella pneumoniae with whole-genome sequencing. Sci Transl Med 4:148ra116. https://doi.org/10.1126/scitranslmed.3004129.

10. Lazaro-Perona F, Sotillo A, Troyano-Hernaez P, Gomez-Gil R, de la VegaBueno A, Mingorance J. 2018. Genomic path to pandrug resistance in a clinical isolate of Klebsiella pneumoniae. Int J Antimicrob Agents 52:713-718. https://doi.org/10.1016/j.jjantimicag.2018.08.012.

11. Sotillo A, Munoz-Velez M, Santamaria ML, Ruiz-Carrascoso G, GarciaBujalance S, Gomez-Gil R, Mingorance J. 2015. Emergence of VIM-1-producing Salmonella enterica serovar Typhimurium in a paediatric patient. J Med Microbiol 64:1541-1543. https://doi.org/10.1099/jmm.0.000170.

12. Pérez-Vazquez M, Oteo-Iglesias J, Sola-Campoy PJ, Carrizo-Manzoni $H$, Bautista V, Lara N, Aracil B, Alhambra A, Martínez-Martínez L, Campos J, Sánchez-Romero I, Orden B, Martínez-Ruiz R, Aznar E, Cercenado E, de la Iglesia P, López-Urrutia L, Salso S, Vicente Saz J, Reyes S, Cobos J, GarcíaPicazo L, Ortega-Lafont M, Megías-Lobón G, Andrés NA, Tarazona ER, Álvarez-García P, Fontanals D, Carranza R, Hernando S, Fe Brezmes M, Ruiz-Velasco LM, Cascales P, Guerrero C, Yolanda G, Rodríguez-Conde I, Saez A. 2019. Characterization of carbapenemase-producing Klebsiella oxytoca in Spain. Antimicrob Agents Chemother 63:2016-2017. https:// doi.org/10.1128/AAC.02529-18.

13. Pérez-Viso $B$, Hernández-García M, Ponce-Alonso M, Morosini MI, RuizGarbajosa P, Del Campo R, Cantón R. 2021. Characterization of carbapenemase-producing Serratia marcescens and whole-genome sequencing for plasmid typing in a hospital in Madrid, Spain (2016-18). J Antimicrob Chemother 76:110-116. https://doi.org/10.1093/jac/dkaa398.

14. Poirel L, Bonnin RA, Nordmann P. 2012. Genetic features of the widespread plasmid coding for the carbapenemase OXA-48. Antimicrob Agents Chemother 56:559-562. https://doi.org/10.1128/AAC.05289-11.

15. Nicolas-Chanoine MH, Mayer N, Guyot K, Dumont E, Pagès JM. 2018. Interplay between membrane permeability and enzymatic barrier leads to antibiotic-dependent resistance in Klebsiella pneumoniae. Front Microbiol 9:1422. https://doi.org/10.3389/fmicb.2018.01422.

16. Hentschke M, Wolters M, Sobottka I, Rohde H, Aepfelbacher M. 2010. ramR mutations in clinical isolates of Klebsiella pneumoniae with reduced susceptibility to tigecycline. Antimicrob Agents Chemother 54:2720-2723. https:// doi.org/10.1128/AAC.00085-10.
17. Bialek-Davenet S, Lavigne JP, Guyot K, Mayer N, Tournebize R, Brisse S, Leflon-Guibout V, Nicolas-Chanoine MH. 2015. Differential contribution of $A c r A B$ and OqxAB efflux pumps to multidrug resistance and virulence in Klebsiella pneumoniae. J Antimicrob Chemother 70:81-88. https://doi.org/ 10.1093/jac/dku340.

18. Wan Nur Ismah WAK, Takebayashi Y, Findlay J, Heesom KJ, Avison MB. 2018. Impact of OqxR loss of function on the envelope proteome of Klebsiella pneumoniae and susceptibility to antimicrobials. J Antimicrob Chemother 73:2990-2996. https://doi.org/10.1093/jac/dky293.

19. Baker S, Hardy J, Sanderson KE, Quail M, Goodhead I, Kingsley RA, Parkhill J, Stocker B, Dougan G. 2007. A novel linear plasmid mediates flagellar variation in Salmonella Typhi. PLoS Pathog 3:e59. https://doi.org/10.1371/ journal.ppat.0030059.

20. Candales MA, Duong A, Hood KS, Li T, Neufeld RA, Sun R, McNeil BA, Wu L, Jarding AM, Zimmerly S. 2012. Database for bacterial group II introns. Nucleic Acids Res 40:D187-D190. https://doi.org/10.1093/nar/gkr1043.

21. Partridge SR, Hall RM. 2003. The IS 1111 family members IS4321 and IS5075 have subterminal inverted repeats and target the terminal inverted repeats of Tn21 family transposons. J Bacteriol 185:6371-6384. https://doi.org/10.1128/jb.185.21.6371-6384.2003.

22. Nicolas E, Lambin M, Dandoy D, Galloy C, Nguyen N, Oger CA, Hallet B. 2015. The Tn3-family of replicative transposons. Microbiol Spectr 3(4):MDNA3-0060-2014. https://doi.org/10.1128/microbiolspec.MDNA3 -0060-2014.

23. Farmer JJ, III, Davis BR, Hickman-Brenner FW, McWhorter A, HuntleyCarter GP, Asbury MA, Riddle C, Wathen-Grady HG, Elias C, Fanning GR. 1985. Biochemical identification of new species and biogroups of Enterobacteriaceae isolated from clinical specimens. J Clin Microbiol 21:46-76. https://doi.org/10.1128/JCM.21.1.46-76.1985.

24. Wyres KL, Holt KE. 2016. Klebsiella pneumoniae population genomics and antimicrobial-resistant clones. Trends Microbiol 24:944-956. https://doi .org/10.1016/j.tim.2016.09.007.

25. Quainoo S, Coolen JPM, van Hijum S, Huynen MA, Melchers WJG, van Schaik W, Wertheim HFL. 2017. Whole-genome sequencing of bacterial pathogens: the future of nosocomial outbreak analysis. Clin Microbiol Rev 30:1015-1063. https://doi.org/10.1128/CMR.00016-17.

26. Jousset AB, Bonnin RA, Rosinski-Chupin I, Girlich D, Cuzon G, Cabanel N, Frech H, Farfour E, Dortet L, Glaser P, Naas T. 2018. A 4.5-year within-patient evolution of a colistin-resistant Klebsiella pneumoniae carbapenemase-producing K. pneumoniae sequence type 258. Clin Infect Dis 67:1388-1394. https://doi.org/10.1093/cid/ciy293.

27. Mathers AJ, Stoesser N, Sheppard AE, Pankhurst L, Giess A, Yeh AJ, Didelot X, Turner SD, Sebra R, Kasarskis A, Peto T, Crook D, Sifri CD. 2015. Klebsiella pneumoniae carbapenemase (KPC)-producing $K$. pneumoniae at a single institution: insights into endemicity from whole-genome sequencing. Antimicrob Agents Chemother 59:1656-1663. https://doi.org/10.1128/AAC.04292-14.

28. Stoesser N, Giess A, Batty EM, Sheppard AE, Walker AS, Wilson DJ, Didelot X, Bashir A, Sebra R, Kasarskis A, Sthapit B, Shakya M, Kelly D, Pollard AJ, Peto TE, Crook DW, Donnelly P, Thorson S, Amatya P, Joshi S. 2014. Genome sequencing of an extended series of NDM-producing Klebsiella pneumoniae isolates from neonatal infections in a Nepali hospital characterizes the extent of community- versus hospital-associated transmission in an endemic setting. Antimicrob Agents Chemother 58:7347-7357. https://doi.org/10.1128/AAC.03900-14.

29. Santos-Lopez A, Marshall CW, Scribner MR, Snyder DJ, Cooper VS. 2019. Evolutionary pathways to antibiotic resistance are dependent upon environmental structure and bacterial lifestyle. Elife 8:e47612. https://doi.org/ 10.7554/eLife.47612.

30. Pitart C, Solé M, Roca I, Fàbrega A, Vila J, Marco F. 2011. First outbreak of a plasmid-mediated carbapenem-hydrolyzing OXA-48 beta-lactamase in Klebsiella pneumoniae in Spain. Antimicrob Agents Chemother 55:4398-4401. https://doi.org/10.1128/AAC.00329-11.

31. Matsumura Y, Peirano G, Bradford PA, Motyl MR, DeVinney R, Pitout JDD. 2018. Genomic characterization of IMP and VIM carbapenemase-encoding transferable plasmids of Enterobacteriaceae. J Antimicrob Chemother 73:3034-3038. https://doi.org/10.1093/jac/dky303.

32. Rutherford JC. 2014. The emerging role of urease as a general microbial virulence factor. PLoS Pathog 10:e1004062. https://doi.org/10.1371/journal .ppat.1004062.

33. Eaton KA, Brooks CL, Morgan DR, Krakowka S. 1991. Essential role of urease in pathogenesis of gastritis induced by Helicobacter pylori in gnotobiotic piglets. Infect Immun 59:2470-2475. https://doi.org/10.1128/IAI.59.7 .2470-2475.1991. 
34. De Koning-Ward TF, Robins-Browne RM. 1995. Contribution of urease to acid tolerance in Yersinia enterocolitica. Infect Immun 63:3790-3795. https://doi.org/10.1128/IAI.63.10.3790-3795.1995.

35. Rózalski A, Sidorczyk Z, Kotełko K. 1997. Potential virulence factors of Proteus bacilli. Microbiol Mol Biol Rev 61:65-89. https://doi.org/10.1128/.61.1 .65-89.1997.

36. Benoit SL, Schmalstig AA, Glushka J, Maier SE, Edison AS, Maier RJ. 2019. Nickel chelation therapy as an approach to combat multi-drug resistant enteric pathogens. Sci Rep 9:13851. https://doi.org/10.1038/s41598-019 $-50027-0$.

37. Maroncle N, Rich C, Forestier C. 2006. The role of Klebsiella pneumoniae urease in intestinal colonization and resistance to gastrointestinal stress. Res Microbiol 157:184-193. https://doi.org/10.1016/j.resmic.2005.06.006.

38. Gorrie CL, Mirceta M, Wick RR, Judd LM, Wyres KL, Thomson NR, Strugnel RA, Pratt NF, Garlick JS, Watson KM, Hunter PC, McGloughlin SA, Spelman DW, Jenney AWJ, Holt KE. 2018. Antimicrobial-resistant Klebsiella pneumoniae carriage and infection in specialized geriatric care wards linked to acquisition in the referring hospital. Clin Infect Dis 67:161-170. https://doi .org/10.1093/cid/ciy027.

39. Clinical and Laboratory Standards Institute (CLSI). 2016. Performance standards for antimicrobial susceptibility testing, twenty-seventh infor mational supplement, M100-S25 edition. Clinical and Laboratory Standards Institute, Wayne, PA.

40. Bankevich A, Nurk S, Antipov D, Gurevich AA, Dvorkin M, Kulikov AS, Lesin VM, Nikolenko SI, Pham S, Prjibelski AD, Pyshkin AV, Sirotkin AV, Vyahhi N, Tesler G, Alekseyev MA, Pevzner PA. 2012. SPAdes: a new genome assembly algorithm and its applications to single-cell sequencing. J Comput Biol 19:455-477. https://doi.org/10.1089/cmb.2012.0021.

41. Chin CS, Alexander DH, Marks $P$, Klammer AA, Drake J, Heiner C, Clum A, Copeland A, Huddleston J, Eichler EE, Turner SW, Korlach J. 2013. Nonhybrid, finished microbial genome assemblies from long-read SMRT sequencing data. Nat Methods 10:563-569. https://doi.org/10.1038/nmeth.2474.

42. Koren S, Walenz BP, Berlin K, Miller JR, Bergman NH, Phillippy AM. 2017 Canu: scalable and accurate long-read assembly via adaptive k-mer weighting and repeat separation. Genome Res 27:722-736. https://doi .org/10.1101/gr.215087.116.

43. Deatherage DE, Barrick JE. 2014. Identification of mutations in laboratory-evolved microbes from next-generation sequencing data using breseq. Methods Mol Biol 1151:165-188. https://doi.org/10.1007/978-1 -4939-0554-6_12.

44. Seemann T. 2014. Prokka: rapid prokaryotic genome annotation. Bioinformatics 30:2068-2069. https://doi.org/10.1093/bioinformatics/btu153.
45. Wyres KL, Nguyen TNT, Lam MMC, Judd LM, van Vinh Chau N, Dance DAB, Ip M, Karkey A, Ling CL, Miliya T, Newton PN, Lan NPH, Sengduangphachanh A, Turner P, Veeraraghavan B, Vinh PV, Vongsouvath M, Thomson NR, Baker S, Holt KE. 2020. Genomic surveillance for hypervirulence and multi-drug resistance in invasive Klebsiella pneumoniae from South and Southeast Asia. Genome Med 12:11. https://doi.org/10.1186/s13073-019-0706-y.

46. Bortolaia V, Kaas RS, Ruppe E, Roberts MC, Schwarz S, Cattoir V, Philippon A, Allesoe RL, Rebelo AR, Florensa AF, Fagelhauer L, Chakraborty T, Neumann B, Werner G, Bender JK, Stingl K, Nguyen M, Coppens J, Xavier BB, Malhotra-Kumar S, Westh $\mathrm{H}$, Pinholt M, Anjum MF, Duggett NA, Kempf I, Nykäsenoja S, Olkkola S, Wieczorek K, Amaro A, Clemente L, Mossong J, Losch S, Ragimbeau C, Lund O, Aarestrup FM. 2020. ResFinder 4.0 for predictions of phenotypes from genotypes. J Antimicrob Chemother 75:3491-3500. https://doi.org/10.1093/jac/dkaa345.

47. Carattoli A, Zankari E, Garcia-Fernandez A, Voldby Larsen M, Lund O, Villa L, Moller Aarestrup F, Hasman H. 2014. In silico detection and typing of plasmids using PlasmidFinder and plasmid multilocus sequence typing. Antimicrob Agents Chemother 58:3895-3903. https://doi.org/10.1128/ AAC.02412-14.

48. Almeida A, Villain A, Joubrel C, Touak G, Sauvage E, Rosinski-Chupin I, Poyart C, Glaser P. 2015. Whole-genome comparison uncovers genomic mutations between group B streptococci sampled from infected newborns and their mothers. J Bacteriol 197:3354-3366. https://doi.org/10 $.1128 / J B .00429-15$.

49. Sayers EW, Karsch-Mizrachi I. 2016. Using GenBank. Methods Mol Biol 1374:1-22. https://doi.org/10.1007/978-1-4939-3167-5_1.

50. Treangen TJ, Ondov BD, Koren S, Phillippy AM. 2014. The Harvest suite for rapid core-genome alignment and visualization of thousands of intraspecific microbial genomes. Genome Biol 15:524. https://doi.org/10.1186/ s13059-014-0524-x

51. Letunic I, Bork P. 2019. Interactive Tree Of Life (iTOL) v4: recent updates and new developments. Nucleic Acids Res 47:W256-W259. https://doi .org/10.1093/nar/gkz239.

52. O'Toole GA, Kolter R. 1998. Initiation of biofilm formation in Pseudomonas fluorescens WCS365 proceeds via multiple, convergent signalling pathways: a genetic analysis. Mol Microbiol 28:449-461. https://doi.org/10 .1046/j.1365-2958.1998.00797.x.

53. Favre-Bonte S, Joly B, Forestier C. 1999. Consequences of reduction of Klebsiella pneumoniae capsule expression on interactions of this bacterium with epithelial cells. Infect Immun 67:554-561. https://doi.org/10 .1128/IAI.67.2.554-561.1999. 